\title{
Digital holographic microscopy for diabetes screening
}

\section{Ana Doblas, Manuel Martínez-Corral, and Genaro Saavedra}

A digital holographic microscope operating in telecentric mode could be used to diagnose diabetes and evaluate long-term glycemic control in patients with diabetes.

Transparent objects, such as live biological cells, are difficult to observe and quantify using traditional light microscopes owing to lack of contrast. However, recent advances in quantitative phase imaging (QPI) of transparent microscopic specimens using digital holographic microscopy (DHM) have facilitated observation of unstained living cells in ways that were previously impossible. This type of microscopy relies on the different optical densities of the specimen and surrounding medium, producing a difference in phase between the light passing through the specimen and the light passing through the medium, ultimately generating an interference pattern. In DHM, only a single recorded image is required to obtain the fringe pattern resulting from the interference between a reference wave and the image of the wavefront diffracted by the specimen. From the pattern, phase changes introduced by the specimen are easily retrieved to produce a quantitative phase map. These phase changes encode the information about the refractive index and thickness of the specimen and as a result, QPI-DHM can be used to analyze illnesses in which the refractive index and/or morphology of cells or tissues are distorted. ${ }^{1-4}$

The application of QPI-DHM to medical diagnosis relies on the high accuracy of the phase map measurements. The optical capture and subsequent computational processing both have a significant role in this hybrid technique. However, our research group has recently demonstrated that the optical capture stage controls the accuracy of QPI-DHM. ${ }^{5-7}$ Specifically, we showed that QPI-DHM operating in telecentric mode-producing a $2 \mathrm{D}$ image of a 3D object-provides very accurate diffraction-limited quantitative phase images with minimal post-processing. This accuracy can be very useful for the diagnosis of different diseases and for specimen identification. Here, we show how this type of microscopy can be used in the diagnosis of diabetes

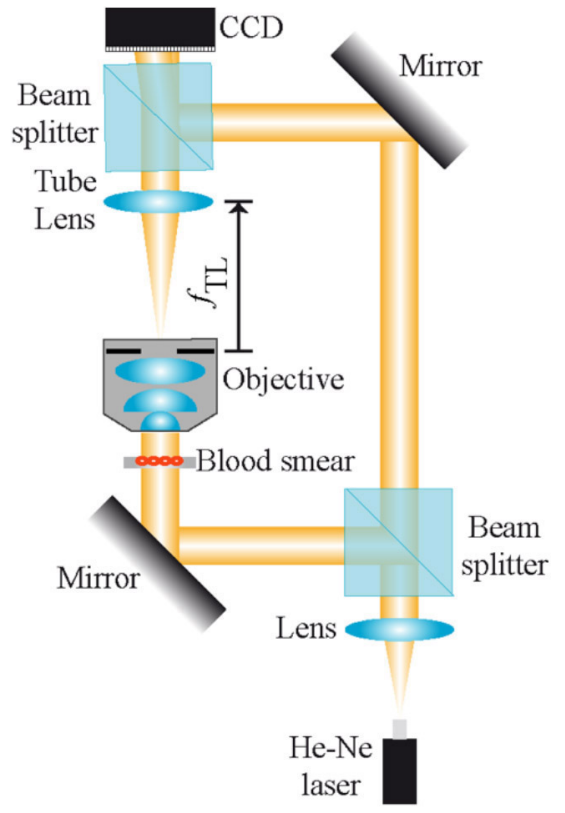

Figure 1. Schematic of the experimental setup for diabetes screening. The digital holographic microscope operates in telecentric mode: the aperture stop of the objective is placed at the front focal plane of the tube lens. $f_{T L}$ : Focal length of the tube lens. He-Ne: Helium-neon.

mellitus (DM), using a simple procedure that requires a small amount of blood.

Telecentric DHM has a potential application as a technique for the screening of $\mathrm{DM}^{8}{ }^{8}$ which is undoubtedly one of the most challenging health problems of this century. Assessment and control of hyperglycemic states is one of the main methods of minimizing the risk of developing the medical complications associated with DM. Currently, the gold standard method to evaluate hyperglycemia in patients with DM and obtain long-term glycemic control is the determination of glycated hemoglobin $(\mathrm{HbA} 1 \mathrm{c})$ concentration. This measurement is usually made by high-performance liquid chromatography (HPLC), 
a complex chemical process requiring the extraction of blood from the antecubital vein. Telecentric DHM can reduce this complexity by imaging phase maps of red blood cells (RBCs).

To evaluate changes to the phase maps of people with $\mathrm{DM}$, we measured phase maps of RBC samples from a group of healthy controls and from patients with DM who were being treated with insulin injections. For each person, we prepared a sample by smearing a capillary blood drop on a glass slide. Each slide was placed in the optical object path of our telecentric digital holographic microscope (see Figure 1). After applying the reconstruction algorithm, we obtained the phase map for each person. Figure 2 shows the phase maps corresponding to one control and one patient with DM. The phase images are pseudocolored so the differences between the phase maps of the healthy control and the patient with DM can be easily visualized. The phase maps clearly show the phase values of diabetic RBCs are significantly higher than those obtained from the healthy person, indicating a difference in the refractive index and/or morphology of the RBCs.

Once the blood smears were imaged, we obtained a mean phase value for each participant by averaging the central phase values of 20 RBCs. Figure 3 (top) shows histograms of the RBC phases from healthy individuals and patients with DM. Again, we observe that the phase values of healthy people were significantly smaller than those of participants with DM: the mean phase values were $3.15 \pm 0.13 \mathrm{rad}$ and $3.72 \pm 0.15 \mathrm{rad}$, respectively. Notably, the phase values of the two groups were clearly separated: the phase values are 2.94-3.30rad for healthy RBCs and 3.51-4.01rad for DM RBCs. From this gap, we can conclude that phase values above 3.40rad correspond to patients with DM.

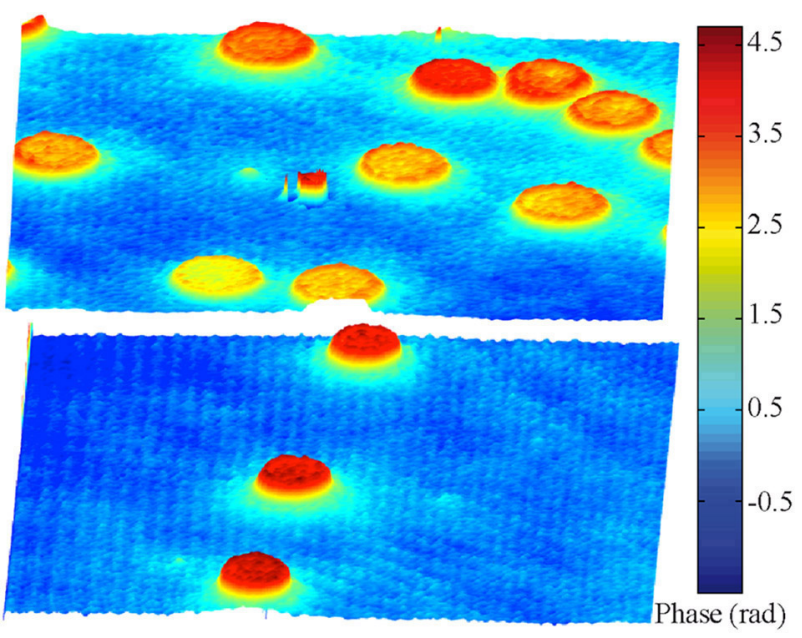

Figure 2. Pseudocolored phase maps of healthy red blood cells (RBCs) (top) and diabetic RBCs (bottom). The image area is $63.5 \times 63.5 \mu \mathrm{m}^{2}$.
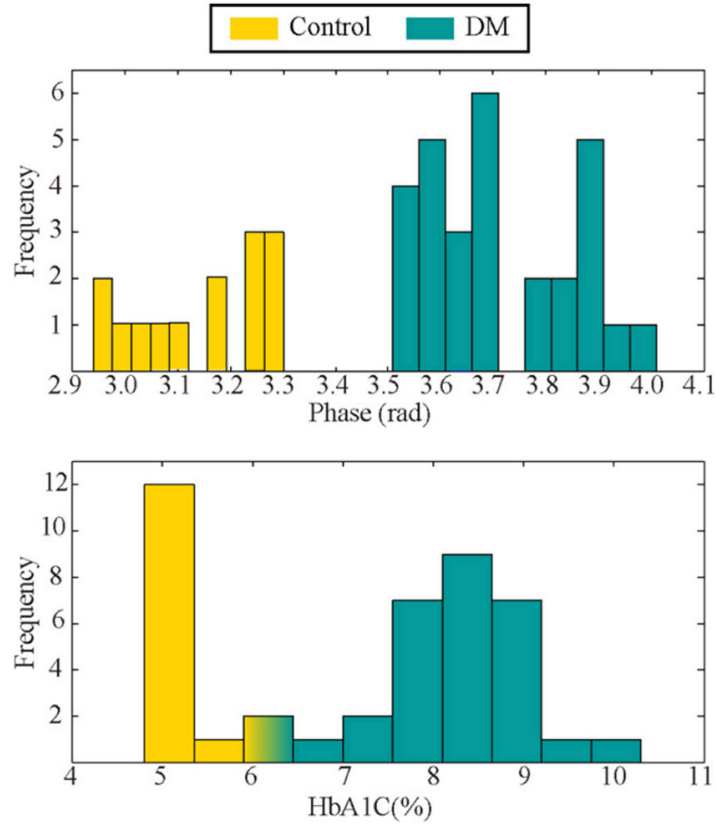

Figure 3. Histograms of mean phase values (top) and glycated hemoglobin (HbA1c) (bottom) of the healthy control group (yellow) and the participants with diabetes mellitus (DM) (turquoise).

As a final test to validate telecentric DHM as a technique to screen for diabetes, we compared our phase values with the $\mathrm{HbA} 1 \mathrm{c}$ values obtained using the standard HPLC method: see Figure 3 (bottom). The correlation coefficient measured between these two distributions was 0.739 . This high correlation coefficient and the clear separation of phase measurements between the two populations confirms that DHM could potentially be used to diagnose diabetes and to evaluate longterm glycemic control in patients with diabetes.

In summary, we have shown that the quantitative phase maps provided by telecentric DHM could be used for the diagnosis of diabetes, and to evaluate long-term glycemic control in patients with diabetes. The main advantages of this technique are that it is minimally invasive, it can be performed at any time, and the phase measurements are rapidly obtained. Our future work in this field will include the experimental implementation of a DHM module in a conventional microscope, and the investigation of further applications of telecentric DHM.

This work was supported in part by the Ministry of Economy and Competitiveness (Spain) through grant DPI 2015-66458-C2-1-R, and by the Generalitat Valenciana (Spain) through grant PROMETEOII/2014/072.

Continued on next page 


\section{Author Information}

\section{Ana Doblas}

Department of Physics and Astronomy

University of North Carolina (UNC)

Chapel Hill, NC

Ana Doblas received her MSc and $\mathrm{PhD}$ in physics from the University of Valencia in 2011 and 2015, respectively. She currently holds a postdoctoral contract at UNC. Her main research interest is $3 \mathrm{D}$ imaging systems.

\section{Manuel Martínez-Corral and Genaro Saavedra \\ Department of Optics \\ University of Valencia \\ Burjassot, Valencia, Spain}

Manuel Martínez-Corral is a full professor of optics and co-leader of the 3D Imaging and Display Laboratory. He received a $\mathrm{PhD}$ in physics from the University of Valencia in 1993. He became an SPIE Fellow in 2010.

Genaro Saavedra received a $\mathrm{BSc}$ and $\mathrm{PhD}$ in physics from the University of Valencia in 1990 and 1996, respectively. His $\mathrm{PhD}$ research was honored by the university with an Extraordinary Award. He is currently an associate professor and has been working at the 3D Display and Imaging Laboratory since 1999. His current research interests are optical diffraction, integral imaging, 3D high-resolution optical microscopy, and phase space representation of scalar optical fields.

\section{References}

1. H. S. Byun, T. R. Hillman, J. M. Higgins, M. Diez-Silva, Z. Peng, M. Dao, R. R. Dasari, S. Suresh, and Y. K. Park, Optical measurement of biomechanical properties of individual erythrocytes from a sickle cell patient, Acta Biomater. 8, pp. 4130-4138, 2012. 2. Y. K. Park, M. Diez-Silva, G. Popescu, G. Lykotrafitis, W. Choi, M. S. Feld, and S. Suresh, Refractive index maps and membrane dynamics of human red blood cells parasitized by Plasmodium falciparum, Proc. Nat'1 Acad. Sci. USA 105 (37), pp. 13730 13735, 2008.

3. N. Pavillon, J. Kühn, C. Moratal, P. Jourdain, C. Depeursinge, P. J. Magistretti, and P. Marquet, Early cell death detection with digital holographic microscopy, PLoS ONE 7, p. e30912, 2012

4. S. Sridharan, V. Macias, K. Tangella, A. Kajdacsy-Balla, and G. Popescu, Prediction of prostate cancer recurrence using quantitative phase imaging, Sci. Rep. 5, p. 9976, 2015.

5. A. Doblas, E. Sánchez-Ortiga, M. Martínez-Corral, G. Saavedra, P. Andrés, and J. Garcia-Sucerquia, Shift-variant digital holographic microscopy: inaccuracies in quantitative phase imaging, Opt. Lett. 38, pp. 1352-1354, 2013.

6. A. Doblas, E. Sánchez-Ortiga, M. Martínez-Corral, G. Saavedra, and J. GarciaSucerquia, Accurate single-shot quantitative phase imaging of biological specimens with telecentric digital holographic microscopy, J. Biomed. Opt. 19, p. 046022, 2014. doi:10.1117/1.JBO.19.4.046022

7. E. Sánchez-Ortiga, A. Doblas, G. Saavedra, M. Martínez-Corral, and J. GarciaSucerquia, Off-axis digital holographic microscopy: practical design parameters for operating at diffraction limit, Appl. Opt. 53, pp. 2058-2066, 2014.

8. A. Doblas, E. Roche, F. J. Ampudia-Blasco, M. Martínez-Corral, G. Saavedra, and J. Garcia-Sucerquia, Diabetes screening by telecentric digital holographic microscopy, J. Microsc. 261, pp. 285-290, 2016. 